\title{
A Comparative Study between Duct-to-Mucosa and Invagination Technique for Reconstruction after Pancreaticoduodenectomy: Aprospective Study
}

\author{
Mohamed El Serafe, MD; Ahmed Hussein Abd El hafiz, MD; \\ Mohamed Shaaban Khalifa, MD.
}

\author{
Department of General Surgery, Ain Shams University, Cairo, Egypt.
}

Background: The pancreaticojejunostomy has notoriously been known to carry a high rate of operative complications,morbidity, and mortality mainly due to anastomotic leak and ensuing septic complications.

Patients and methods: From January 2012 to October 2015, we presented a prospective study which included 24 patients who underwent pancreaticoduodenectomy (PD) operation through either Whipple resection or modified Whipple(pylorus-preserving).Patients were reviewed and divided into 2 groups $(A, B)$ according to the type of pancreaticojejunostomy $(P J)$, (invagination vs duct-to-mucosa).

Results: 12 patients in each group were operated on: Group A patients had invagination technique for $P J$, while all the 12 patients in group B had duct-to-mucosa anastomotic technique for PJ. 1 (8.3\%)case in group A developed pancreatic fistula (PF), while $3(25 \%)$ cases in group $B$ developed $P F$, and 1 case (8.3\%) in group A had mild anastomotic leak which was managed conservatively,while 3 cases (25\%) in group B developed moderate to severe anastomotic leak with intra-abdominal collection which required CT-guided percutaneous drainage and operative intervention. Average age was (mean $\pm S D)=(55 \pm 12)$, average operative time was (245 \pm 75$)$ min.

Conclusion: PF after PD represents a critical trigger of potentially life-threatening complications. Although the best method for dealing with the pancreatic stump after PD remains controversial, recent reports described the invagination technique todecrease the rate of PF significantly compared to the duct-to-mucosa technique. Our results appeared to be closely related to the published literature.

Key words: Pancreatic fistula (PF), pancreaticodudenectomy (PD), pancreaticojejunostomy (PJ), invagination technique, duct-to-mucosa technique.

\section{Introduction:}

Theindicationsof pancreaticodudenectomy (PD) have expanded to encompass a broad spectrum of periampullary tumors including both benign and malignant lesions, chronic pancreatitis, and occasionally trauma. During the last decade, although the rate of operative mortality significantly decreased after PD, the incidence of post-operative morbidity still remains high.. ${ }^{1,2}$

The occurance of $\mathrm{PF}$ is a critical trigger of life-threatening complications such as intra-abdominal abscess and hemorrhage, ${ }^{3}$ whichis also potentially associated with markedly prolonged hospitalization. Most of the large PD series have reported rates of PF of over $10 \% .4,5$ Risk factors for PF depends upon: 1) general patient factors including age, ${ }^{6}$ sex, ${ }^{7} \mathrm{DM},{ }^{8}$ and nutrition; ${ }^{9}$ 2) diseaserelated factors,including pancreatic duct size,pancreatic texture,and pathology; 10 3 ) procedure-related factors including, blood loss, operative time, ${ }^{11}$ and anastomotic technique. ${ }^{12}$ Among the risk factors, the most 
important might be the texture of the remnant pancreas. 13

Indeed, despite an occurance rate of $\mathrm{PF}$ of $5 \%$ in cases of hard pancreatic tissue, the rate rises to nearby $20 \%$ in cases of soft pancreatic tissue. ${ }^{14}$ The risk of developing a $\mathrm{PF}$ is significantly associated with the final histopathological diagnosis of the resected specimen, with lower risk in adenocarcinoma, and high risk in cystic neoplasm, or diseases originating from the bile duct. ${ }^{15}$ This is also because pancreatic malignancy usually causes main pancreatic duct dilatation which also occurs in chronic pancreatitis; therefore, a fibrotic hard remnant pancreas and an enlarged duct are easily anastomosed, whereas soft pancreas remains at risk of developing pancreatic fistula due to its fragility and its secretion of large amounts of pancreatic juice. ${ }^{16}$ Surgical technique might be one improvable aspect of PD that can reduce the pancreatic leakage rate; it is critical in the management of the pancreatic remnant because of the various methods used by surgeons. Methods of reconstruction which are used between pancreatic stump and intestine include end-to-side with or without duct-to-mucosa anastomosis, end-to-end invagination technique. ${ }^{17}$

\section{Patients and methods:}

From January 2012 to October 2015, we reviewed 24 consecutive patients, who underwent pancreaticodudenectomy (PD), all patients were studied and evaluated. Patient demographic data,full history and clinical examination, full preoperative laboratory and radiological investigations were obtained. The patients had either a classic Whipple resection $(n=16)$ (66.7\%), or pyloruspreserving $\mathrm{PD}(\mathrm{n}=8)$ (33.3\%), according to the decision of the attending surgeon. All patients were thoroughly informed about the procedure,and signed consent was obtained. Preoperative evaluation consisted of an extensive imaging workup including computed tomography (CT), magnetic resonance imaging (MRI), and magnetic resonance cholangio-pancreatography (MRCP), to rule out metastatic disease or encasement of superior mesenteric vessels (SMV). Transduodenal fine needle biopsy (FNA), and endoscopic biliary stenting were occasionally used whenever required, the final assessment of the respectability of the malignant tumor was made intraoperatively, based upon findings such as liver metastases not identified preoperatively and involvement or encasement of SMV or portal vein. All patients received preoperative antibiotic prophylaxis, and low molecular weight heparin was administered for 4 weeks. None of the patients received octreotide analogue preoperatively or postoperatively. The pancreatico-jejunostomy anastomotic technique used was either (A) invagination or (B) duct-to-mucosa,based on these variables ,patients were divided into 2 groups, group A had invagination technique, and group B had duct-to-mucosa anastomotic technique. Epidemiological, operative, and histological data are presented in Table (1).

\section{Surgical technique:}

After completion of the standard PD and thorough hemostasis, the jejunum was transected at about $20 \mathrm{~cm}$ from ligament of Treitz's, reflected upwards through an incision in the transverse mesocolon, and anastomosed end-to-side with the choledochus by one layer of interrupted 4-0 polydioxanone surgical sutures. At $20 \mathrm{~cm}$ distal to the biliary anastomosis, the jejunum was interrupted, and the end of the pancreas inserted into the bowel by means of either one of the following 2 techniques, regardless of the diameter of the pancreatic duct, (a) the invagination technique created with 2 rows of continuous or interrupted sutures done via Prolene 3-0 (Ethicon), between the cut edges of the jejunum (seromuscular layer) and the pancreatic parenchyma, directing the end of the pancreas to invaginate into the jejunum, and (b) the end-to-side duct-to-mucosa PJ established with the use of interrupted 5-0 or 6-0 PDS (Ethicon) sutures between the pancreatic duct and the mucosa of the jejunum, and reinforced by seromuscular sutures from the jejunum to the cutting edge of the pancreatic parenchyma. At 20 
cm distal to the pancreatico-jejunostomy (PJ), the jejunum was anastomosed to the gastric pouch in an end-to-side fashion (gastrojejunostomy), or to the duodenal loop in case of pylorus-preserving resection (duodenojejunostomy). The alimentary tract reconstruction was accomplished via Rouxen-Y enteroenterostomy, finally a closed suction tube drain was placed near the PJ anastomosis and brought out through the right lateral abdominal wall Figures $(\mathbf{1 , 2 , 3}) .{ }^{18}$

\section{Perioperative management-Data collection:}

All patients received a standard perioperative management. Details of surgical procedure and complications were recorded,drain fluid volume and amylase levels were monitored daily, nasogastric (Ryle) tube was removed, and feeding was introduced as soon as adequate gastric emptying was re-established. The drain tube was removed 6 to 10 days post operatively,provided that amylase levels in the drainage fluid did not exceed serum levels by more than 3 times. When intra-abdominal collections were suspected, an abdominal CT was done and percutaneous aspiration was carried out for fluid culture and biochemical analysis. A percutaneous tube for drainage under CT guidance was inserted when pancreatic collection reoccurred or an intraabdominal abscess developed.

A pancreatic fistula (PF) as a result of pancreatic leakage was defined as a drainage volume of more than $50 \mathrm{ml}$ after the $8^{\text {th }}$ postoperative day,with an amylase level greater than $1000 \mathrm{IU} / \mathrm{ml}$, or greater than 3 times the serum amylase level. In accordance to the definition of the International Study Group of Pancreatic Fistula. ${ }^{19}$ When a diagnosis of a pancreatic fistula was established, the tube remained in place until the fistula volume was reduced to a level less than 50 $\mathrm{ml} /$ day. The severity of pancreatic leakage was graded as: (1) grade A, when it subsides without intervention; (2) grade B, when a non surgical intervention was applied to resolve the complication by using percutaneous CTguided drainage, and appropriate antibiotic therapy; and (3) grade C, when operative intervention was required. ${ }^{20}$

\section{Results:}

In this prospective study, 24 patients presented by pancreatic lesions in the form of malignant tumors, or benign conditions such as chronic pancreatitis. All the patients were subjected to pancreaticodudenectomy (PD) via Whipple resection or pylorus-preserving operation. All the patients had full laboratory and radiological investigations to assess operability , and metastatic disease.Whipple resection was performed in 18 patients (75\%), while pylorus-preserving procedure was performed in 6 patients (25\%), according to the procedure chosen for $\mathrm{PJ}$ anastomosis,the 24 patients were classified into 2 groups (A,B). Group A involved 12 patients underwent $\mathrm{PJ}$ anastomosis via invagination technique,while group B (12) patients underwent $\mathrm{PJ}$ anastomosis via duct-to-mucosa technique,average age of the patients was (55 \pm 12 ) years, average operative time was (245 \pm 75$)$ min, with an average intraoperative blood loss (750 $\pm 200 c c)$, the average hospital stay (12 \pm 2.5 ) days. Total parenteral nutrition (TPN) was instituted in those patients who developed any anastomotic defect and delayed gastric emptying. Both groups of patients were comparable regarding epidemiological, histological, and intraoperative data Table (1).

As regard pancreatic leakage incidence, 1 patient (8.3\%) in group A developed pancreatic anastomotic leakage with mild intra-abdominal collection which subsided conservatively, while 3 patients (25\%) in group B developed moderate to severe pancreatic leakage, pancreatic leakage incidence in relation to the type of PJ anastomosis is presented in Table (2). The use of invagination technique for $\mathrm{PJ}$ anastomosis was associated with a significantly lower pancreatic leakage rate as compared with the use of duct-to-mucosa technique (8.3\% vs 25\%, $\mathrm{P}=0.035$ ).

Postoperative complications, morbidity, hospitalization, and mortality are presented in Table (3). 3 patients (75\%) in group (B) developed PF, 2 of them had grade B pancreatic fistula which required CT-guided 
percutaneous drainage and antibiotic therapy, the $3^{\text {rd }}$ patient had grade $\mathrm{C}$ pancreatic fistula which required surgical intervention, the disruption of the $\mathrm{PJ}$ anastomosis that was found in this patient was managed by means of stable closure of the jejunal loop, and external drainage of the pancreatic stump. While in group (A), 1 patient only (8.3\%) developed grade A pancreatic fistula, which resolved and closed spontaneously.

The overall morbidity was significantly high in group (B) patients who had duct-tomucosa than those of group (A) who had invagination technique for $\mathrm{PJ}$ anastomosis (the overall morbidity rate in group $\mathrm{A}$ was $33.3 \%$ vs $83.3 \%$ in group $\mathrm{B}, \mathrm{P}=0.0046$ ). The length of hospital stay was significantly increased in group (B) patients, than those of group (A) patients $(\mathrm{P}<0.01)$. The overall mortality rate in our study was $2.8 \%$. Two patients (16.6\%) died in group (B) due to major anastomotic leak and severe intraperitoneal sepsis, while 1 patient (8.3\%) in group (A) died due to massive myocardial infarction. 3 patients (25\%) in group (B) had developed moderate to severe anastomotic dehiscence and leakage, 2 of them were managed via CTguided percutaneous drainage and antibiotic coverage,and the $3^{\text {rd }}$ one needed surgical intervention, and open drainage of intraabdominal abscess. While 1 patient (8.3\%) in group (A) developed minor anastomotic leak and mild collection which was managed conservatively.

\section{Discussion:}

Drainage of the pancreatic remnant to the gastrointestinal tract has been thoroughly investigated, and various techniques have been reported. Despite sporadic reports describing low rates of pancreatic leakage (from 3\% up to $14.3 \%$ ) after pancreaticogastrostomy, no valid conclusion can be drawn regarding its superiority against $\mathrm{PJ}$, and further randomized controlled trials are required. ${ }^{21}$

$\mathrm{PJ}$ is still the most commonly used method of restoring pancreatico-enteric continuity after PD, and its technical improvements are essential to reduce the pancreatic leakage rate. $^{22}$
Regarding anastomotic techniques used for reconstruction between the pancreatic cut surface and the jejunum, both end-toside duct-to-mucosa anastomosis or endto-end invagination techniques have been extensively investigated. Certain reports have shown no clear evidence for or against one particular method of pancreatico-enteric anastomosis. $^{23}$

The choice of pancreatic anastomosis method might be based on individual experience and adherence to basic principles such as good exposure and visualization, fine, non strangulating suture placement, to produce a patent, watertight anastomosis; and preservation of blood supply. Recent reports described the invagination method to decrease the rate of pancreatic fistula significantly compared to the duct-to-mucosa anastomosis. ${ }^{24}$

In our study, the incidence of PF in group (B) patients operated upon via duct-to-mucosa technique, and the incidence of anastomotic dehiscence and leakagewas relatively higher as compared to group A patients who were operated upon via invagination technique, 3 patients (25\%) in group (B) developed PF, compared to 1 patient (8.3\%) in group (A) developed PF, also 3 patients (25\%) in group B developed severe anastomotic leakage and managed via CT-guided percutaneous drainage and operative intervention, in comparison to 1 patient (8.3\%) in group A developed mild anastomotic dehiscence and mild collection managed conservatively.

Mortality rate was higher in group $\mathrm{B}$ $(16.6 \%)(n=2)$ as compared to group A (8.3\%) $(n=1)$, in our study TPN was given to all patients who developed PF and anastomotic leakage.

Ferguson and Wangesteen (1998); were the 1st to report a new interesting experimental technique for PJ anastomosis, directly approximating the jejunal mucosa to the Wirsung duct epithelium, subsequently described by Madden. ${ }^{25}$ Since then, other modifications have been suggested, such as the 2 layers technique, the use of fibrin glue, and the use of ultrasonic shears. ${ }^{26}$

Weagnee with Sikora and Posner (1995), 


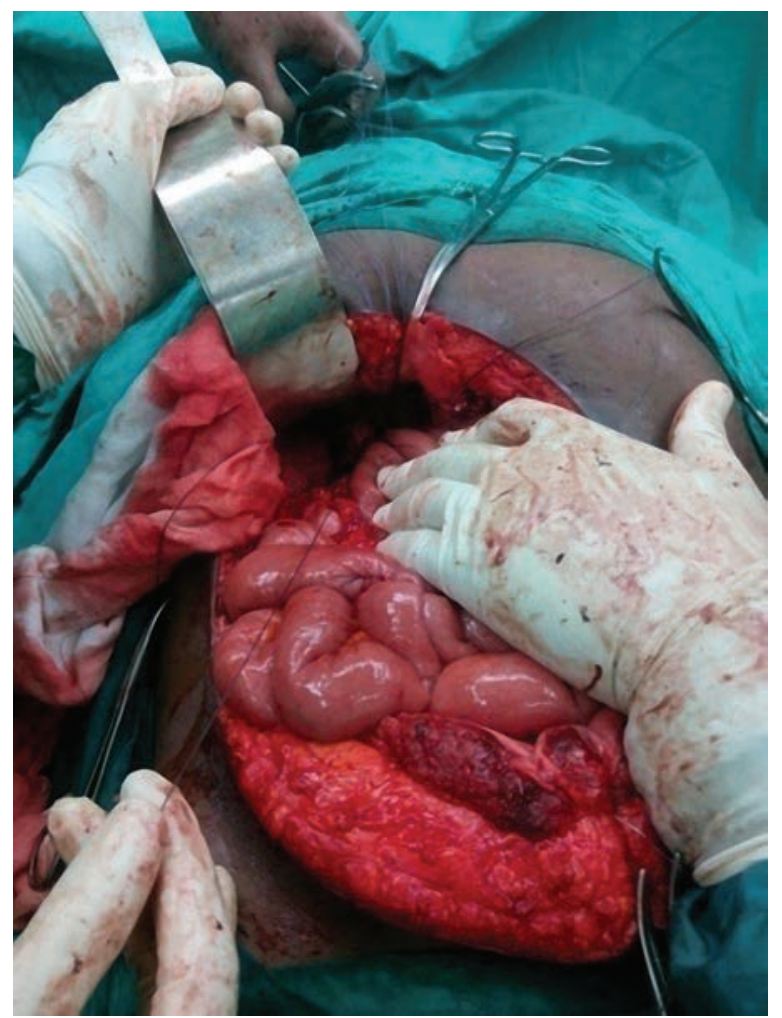

Figure (1): Hepaticojejunostomy.

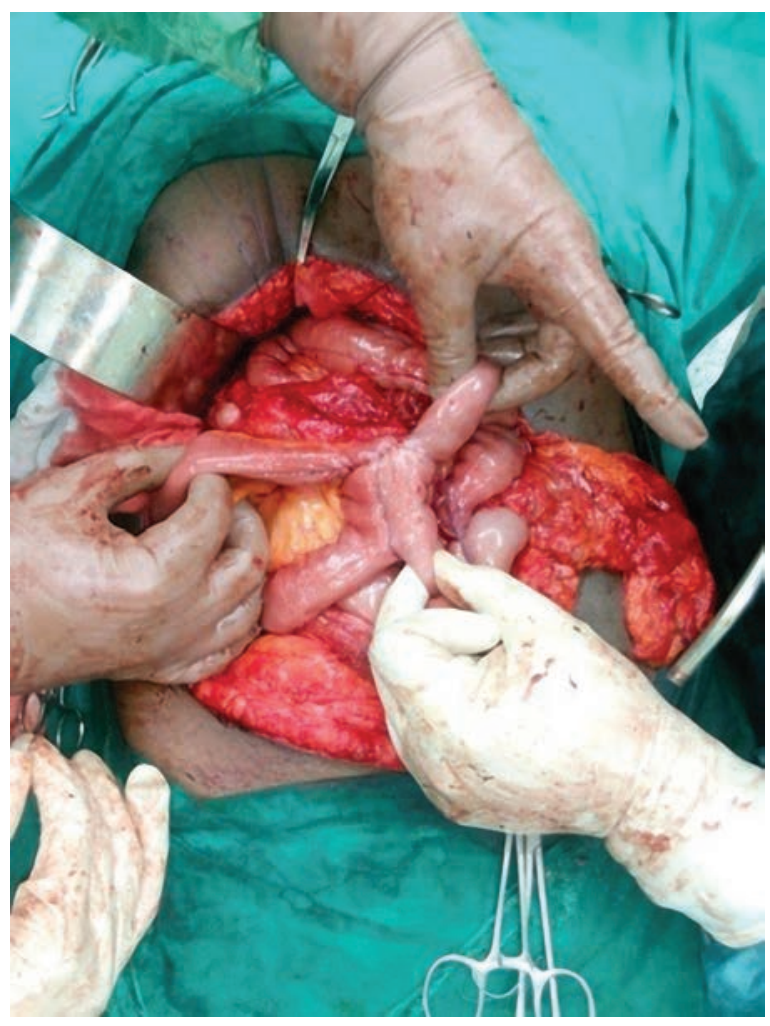

Figure (2): Enteroenterostomy.

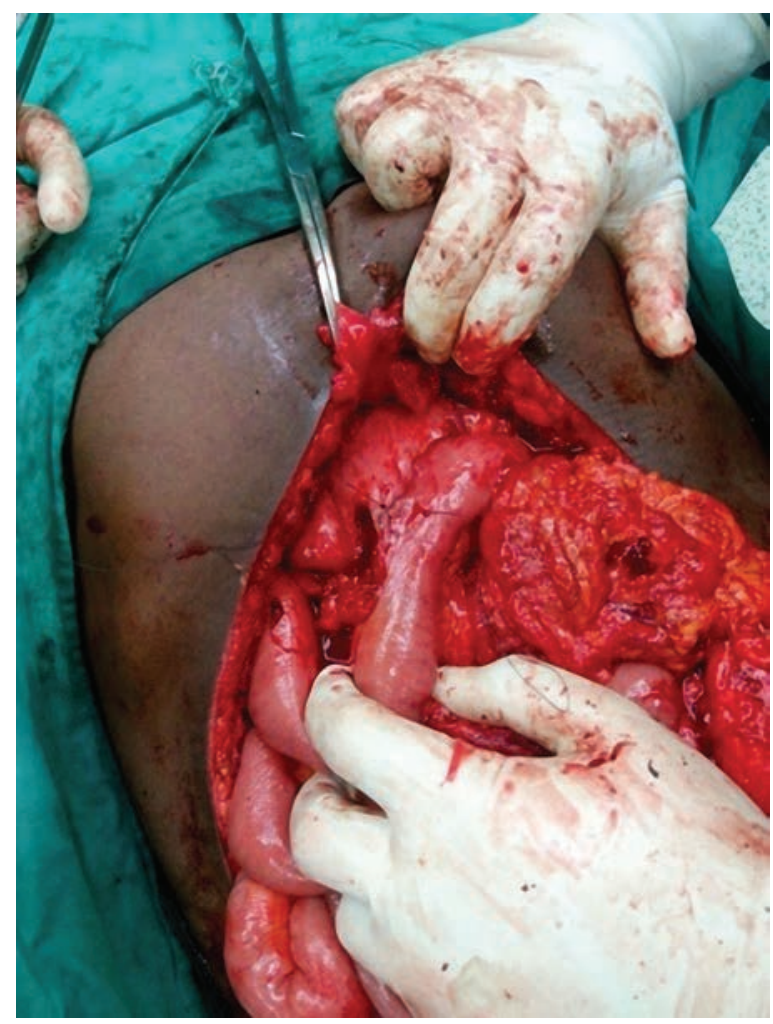

Figure (3): Gastrojejunostomy.

Marcus et al;and Suzuki et al (2002); using the selective duct-to-mucosa technique when the Wirsung duct is dilated $(>5 \mathrm{~mm})$ and in the presence of firm fibrotic pancreas, ${ }^{27}$ whereas the invagination technique appears to be safer and then should be performed in the presence of friable pancreas with nondilated duct, considering the invagination technique for $\mathrm{PJ}$ is always feasible in all situations (small or dilated duct, friable or 
Table (1): Epidemiological, operative, and histological data of patients.

\begin{tabular}{|l|l|l|}
\hline & Group A (invagination) & Group B (duct-to- mucosa) \\
\hline *Number of patients & 12 & 12 \\
\hline *Age of patients & $58 \pm 15$ & $60 \pm 11$ \\
\hline *Sex, male : Female & $10: 2$ & $8: 4$ \\
\hline *Type of PD & & \\
\hline Convential & 8 & 10 \\
\hline Pylorus- preserving & 4 & 2 \\
\hline *Operative data & & \\
\hline Duration of procedure/m & $240 \pm 80$ & $255 \pm 90$ \\
\hline Blood loss/ml & $700 \pm 250$ & $800 \pm 280$ \\
\hline *Pathological diagnosis: & & \\
\hline 1) Pancreatic adenocarcinoma & 7 & 6 \\
\hline 2) Cholangiocarcinoma & 2 & 2 \\
\hline 3) Periampullary carcinoma & 2 & 2 \\
\hline 4) Duodenal carcinoma & - & 1 \\
\hline 5) Chronic pancreatitis & 1 & 1 \\
\hline $\begin{array}{l}\text { 6)Others (duodenal } \\
\text { carcinoid,neuroendocrine) }\end{array}$ & - & - \\
\hline *Tumor staging & & 3 \\
\hline 1) Tumor size & $3.8 \pm 1.2$ & $3.9 \pm 1.1$ \\
\hline 2) Positive LNs & $8 \pm 3.6$ & $9 \pm 3.8$ \\
\hline *Tumor differentiation & & 3 \\
\hline 1) High & 4 & \\
\hline 2) moderate & 1 & \\
\hline 3) Poor & & \\
\hline
\end{tabular}

*Mean \pm SD

Table (2): Comparison of the pancreatic leakage rates in the 2 groups.

\begin{tabular}{|l|l|l|l|}
\hline \multirow{2}{*}{} & \multicolumn{2}{|c|}{ Pancreatic leakage } & \multirow{2}{*}{ P } \\
\cline { 2 - 3 } & \multicolumn{1}{|c|}{ Yes(\%) } & \multicolumn{1}{|c|}{ No } & \\
\hline *Anastomosis & & & \\
\hline Invagination & $1(8.3 \%)$ & 11 & 0.042 \\
\hline Duct-to-mucosa & $3(25 \%)$ & 9 & 0.047 \\
\hline
\end{tabular}

firm, fibrotic pancreas), ${ }^{28}$ it should be the procedure of choice. The duct-to-mucosa technique after PD in case of a normal, non-dilated Wirsung duct is a more time consuming, and demanding technique, necessarily requiring a microsurgical skills, but it can be alternatively adopted in case of an enlarged gland that does not fit well in the jejunum to be invaginated. ${ }^{29,30}$

\section{Conclusion:}

Although the best method for dealing with the pancreatic stump after PD remains controversial, recent reports described that the invagination technique to decrease the rate of $\mathrm{PF}$ significantly compared to the duct-to-mucosa technique, although the low number of patients in our series that did not allow definitive conclusion, more time, and 
Table (3): Postoperative complications, and outcome of patients.

\begin{tabular}{|l|l|l|}
\hline \multicolumn{1}{|c|}{ Postoperative complications } & \multicolumn{1}{c|}{ Group (A) } & Group (B) \\
\hline *Pancreatic fistula & $1(8.3 \%)$ & $3(25 \%)$ \\
\hline$*$ Intra-abdominal collection & $1(8.3 \%)$ & $3(25 \%)$ \\
\hline$*$ Intra-abdominal hemorrhage & - & 1 \\
\hline$*$ GI obstruction & - & - \\
\hline *Delayed gastric emptying & 2 & 4 \\
\hline *Biliary fistula & - & - \\
\hline *Chest infection & 1 & 3 \\
\hline *Pulmonary effusion & 1 & 1 \\
\hline$*$ GI bleeding & - & 1 \\
\hline$*$ Wound infection & 2 & 5 \\
\hline$*$ Overall morbidity rate, \% patients & $4(33.3 \%)$ & $10(83.3 \%)$ \\
\hline$*$ Hospital stay/day & $10.6 \pm 2$ & $15.8 \pm 1.8$ \\
\hline$*$ Mortality & $1(8.3 \%)$ & $2(16.6 \%)$ \\
\hline
\end{tabular}

experience is needed, for more accurate results. ${ }^{31,32}$

Our results encouraged the use of invagination technique as compared to the duct-to-mucosa technique, as it seems to be more save, feasible, and associated with less incidence of postoperative complications, less morbidity, mortality,and shorter hospital stay.

\section{Reference:}

1- Buchler MW, Wagner M, Schmied BM: Changes in morbidity after pancreatic resection; toward the end of completion pancreatectomy. Arch Surg 2003; 138: 1310-1315.

2- Tsuchiya R, Tsunada T, IschidaT: Resection for cancer of the pancreas. The Japanese experience. Baillieres Clin Gastroenterol 2012; 4: 931-939.

3- Neoptolemos JP, Russel RC, BramhallS: Low mortality following resection for pancreatic and periampullary tumors in 1016 patients: UK survey of specialist pancreatic units. UK Pancreatic Cancer Group. Br J Surg 2008; 84: 1370-1376.

4- Kleespies A, Albertsmeir M, Obeidat F: The challenge of pancreatic anastomosis. Langenbecks Arch Surg 2008; 39: 450-456.

5- Bottger TC, JungingerT: Factors influencing morbidity and mortality after pancreaticoduodenectomy; critical analysis of 221 resections. World J Surg 2009; 23:
164-171.

6- Buchler MW, Friess H, Wagner M: Pancreatic fistula after pancreatic head resection. $\mathrm{Br} J$ Surg 2010; 87: 883-889.

7- Gouma DJ, van Geenen RC, van Gulik YM: Rates of complications and deaths after pancreaticoduodenectomy; risk factors and the impact of hospital volume. Ann Surg 2008; 232: 788-795.

8- Sohn TA, Yeo CJ, Cameron JL: Resected adenocarcinoma of the pancreas-616 patients; results, outcomes, and prognostic indicators. J Gastroenterol Surg 2011; 4: 138-146.

9- Wade TP, el-Ghazzawy AG, Virgo KS: The Whipple resection for cancer in U.S. Department of Veterans Affairs Hospital. Ann Surg 2002; 221: 241-248.

10- Halloran CM, Ghareh P, BosonnetL: Complications of pancreatic cancer resection. Dig Surg 2002; 19: 138-146.

11- Yeh TS, Jan YY, Jeng LB: Pancreaticojejunal anastomosis leak after pancreaticoduodenectomy; multivariate analysis of perioperative risk factors. $J$ Surg Res 2005; 67: 119-125.

12- Crippa S, Salvia R, Falconi M: Anastomotic leakage in pancreatic surgery. $\mathrm{Br} J$ Surg 2007; 9: 10-15.

13- Tran K, Van Eijck C, Di Carlo V : Occlusion of the pancreatic duct after pancreaticojejunostomy: A prospective randomized trials. Ann Surg 2012; 236: 422-428.

14- Papardoriston DN, De Agostine H, 
Fortner JG: Ligation of pancreatic duct in pancreatectomy. $\mathrm{Br} J$ Surg 2006; 67: 260-265.

15- Lillemoe KD, Cameron JL, Kim MP: Does fibrin glue sealant decrease the rate of pancreatic fistula after pancreaticoduodenectomy? Results of a prospective randomized trials. $J$ Gastrointest Surg 2004; 8: 766-774.

16- GebhardtC, Stolte M, Schwille PO: Experimental studies on pancreatic duct occlusion with Prolene. Horm Metab Res Suppl 2008; 13: 190-195.

17- Poon RTP, LO SH, Fong D: Prevention of pancreatic anastomotic leakage after pancreaticoduodenectomy. Am J Surg 2003; 183: 42-50.

18- Miedema BW, Sarr MG, van Heerden JA, Nagorney DM: Complications following pancreaticoduodenectomy. Current management. Arch Surg 2010; 127: 450-458.

19- Fernandez del Castillo, Rattner DW, WarshawAL: Standards for pancreatic resection. Arch Surg 2005; 139: 552-563.

20- Crist DW, Cameron JL: Current status of pancreatoduodenectomy for periampullary carcinoma. Hepatogastroenterol 2009; 36: 478-485.

21- Grace PA, Pitt HA, Longmire WP: Pancreatoduodenectomy with pylorus preservation for adenocarcinoma of the head of the pancreas. Br J Surg 2008; 73: 675-682.

22- Bruasch JW, Rossi RL, Watkins E Je: Pyloric and gastric preserving pancreatic resection. Experience with 87 patients. Ann Surg 2002; 204: 411-418.

23- Bassi C, FalconiM, Mollinari E: Duct-to-mucosa versus end-to-side pancreaticojejunostomy reconstruction after pancreaticoduodenectomy: Results of a prospective randomized trial. Surgery 2003; 134: 766-771.

24- Hosotani R, Doi R, Imamura M: Duct-tomucosa pancreaticojejunostomy reduces the risk of pancreatic leakage after pancreaticoduodenectomy. World $J$ Surg 2008; 26: 99-104.

25- Dmitrewski J, Buckels JA: A simple modification of choledochoenterostomy using continuous pull through suture. Surg Gynecol Obstet 2008; 175: 573-578.

26- Marctzell AP, Steiner M: Partial pancreaticoduodenectomy (Whipple procedure) for pancreatic malignancy: Occlusion of a non-anastomosed pancreatic stump with Neoprene injection. World J Surg 2009; 13: 105-111.

27- Sikora SS, Posner M: Management of the pancreatic stump following pancreaticoduodenectomy. Br J Surg 1995; 82: 1590-1597.

28- Ferguson DJ, Wangenstien ON: Experimental anastomosis of the pancreatic duct. Ann Surg 1998; 132: 1066-1074.

29- Madden JL: Technique for pancreaticoduodenectomy. Surg Gynecol Obstet 1994; 118: 246-265.

30- Howard JM: Pancreatojejunostomy: Leakage is a preventable complication of Whipple resection. J Am Coll Surg 1997; 184: 454-457.

31- Marcus SG, Cohen H, Ranson JH: Optimal management of pancreatic remnant after pancreaticoduodenectomy. Ann Surg 1995; 221: 635-645.

32- Suzuki Y, Fujino Y, Tunioka Y: Selection of pancreaticojejunostomy techniques according to pancreatic texture and duct size. Arch Surg 2002; 137: 1044-1047. 